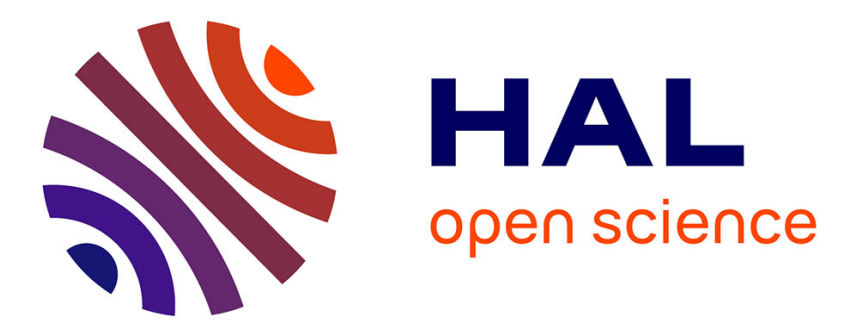

\title{
Hovering of a passive body in an oscillating airflow
}

Stephen Childress, Nicolas Vandenberghe, Jun Zhang

\section{To cite this version:}

Stephen Childress, Nicolas Vandenberghe, Jun Zhang. Hovering of a passive body in an oscillating airflow. Physics of Fluids, 2006, 18, pp.117103. 10.1063/1.2371123 . hal-00114045

\section{HAL Id: hal-00114045 \\ https://hal.science/hal-00114045}

Submitted on 7 Sep 2015

HAL is a multi-disciplinary open access archive for the deposit and dissemination of scientific research documents, whether they are published or not. The documents may come from teaching and research institutions in France or abroad, or from public or private research centers.
L'archive ouverte pluridisciplinaire HAL, est destinée au dépôt et à la diffusion de documents scientifiques de niveau recherche, publiés ou non, émanant des établissements d'enseignement et de recherche français ou étrangers, des laboratoires publics ou privés. 


\title{
Hovering of a passive body in an oscillating airflow
}

\author{
Stephen Childress ${ }^{\text {a) }}$ \\ Applied Mathematics Laboratory, Courant Institute of Mathematical Sciences, New York University, \\ 251 Mercer Street, New York, New York 10012 \\ Nicolas Vandenberghe ${ }^{\text {b) }}$ \\ Applied Mathematics Laboratory, Courant Institute of Mathematical Sciences, New York University, \\ 251 Mercer Street, New York, New York 10012 and IRPHE, Université de Provence, \\ 13384 Marseille, France \\ Jun Zhang ${ }^{\text {) }}$ \\ Department of Physics, New York University, 4 Washington Place, New York, New York 10003 \\ and Applied Mathematics Laboratory, Courant Institute of Mathematical Sciences, New York University, \\ 251 Mercer Street, New York, New York 10012
}

(Received 26 June 2006; accepted 27 September 2006; published online 14 November 2006)

\begin{abstract}
Small flexible bodies are observed to hover in an oscillating air column. The air is driven by a large speaker at frequencies in the range $10-65 \mathrm{~Hz}$ at amplitudes $1-5 \mathrm{~cm}$. The bodies are made of stiffened tissue paper, bent to form an array of four wings, symmetric about a vertical axis. The flapping of the wings, driven by the oscillating flow, leads to stable hovering. The hovering position of the body is unstable under free fall in the absence of the airflow. Measurements of the minimum flow amplitude as a function of flow frequency were performed for a range of self-similar bodies of the same material. The optimal frequency for hovering is found to vary inversely with the size. We suggest, on the basis of flow visualization, that hovering of such bodies in an oscillating flow depends upon a process of vortex shedding closely analogous to that of an active flapper in otherwise still air. A simple inviscid model is developed illustrating some of the observed properties of flexible passive hoverers at high Reynolds number. (c) 2006 American Institute of Physics.
\end{abstract}

[DOI: $10.1063 / 1.2371123$ ]

\section{INTRODUCTION}

This paper is concerned with the mechanisms of hovering flight. Our interest is in the aerodynamics of birds and insects, and also the application of aerodynamic theory to the construction of tiny robotic micro-flyers with hovering capability. Our main object here is to describe an experimental tool that may be useful in this endeavor. For want of a better term, we characterize the device as a "hovering simulator." It is essentially a vertical wind tunnel with an airflow which oscillates upward and downward with no mean component. A passive deformable body placed in this flow can respond by changing its shape. As we shall show, this fact can be exploited to mimic the movements of a hovering insect, with surprising results.

Hovering flight is a special case of locomotion in which there is little or no average movement of the body relative to a fixed point in space. In body coordinates, there is no "ambient uniform stream" as one has in forward flight. Body movements associated with hovering must therefore be conditioned to deal with whatever flow field is created by the hoverer, assuming that the latter is negatively buoyant and thus delivers downward momentum to the fluid on average. This calls for special mechanisms of lift production, or at least substantial modification of the mechanisms used for

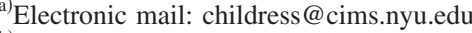

b) Electronic mail: nicolas.vandenberghe@irphe.univ-mrs.fr

${ }^{c)}$ Electronic mail: jun@cims.nyu.edu
}

forward flight. ${ }^{1,2}$ So-called "normal" hovering, such as one sees in hummingbirds, involves back and forth horizontal movements of the wings, which pivot so as to maintain a favorable angle of attack. "This kind of lift production can be partially understood with "quasi-steady" aerodynamic theory, although there are departures from this approximation. ${ }^{4}$ Many insects, however, depend upon fully unsteady aerodynamics, in which the typical flow velocity $U$ is comparable to the wingbeat frequency $f$ times a representative length. ${ }^{1} \mathrm{~A}$ notable example of an unsteady flight mechanism is the "clap and fling" discovered by Weis-Fogh, ${ }^{3}$ but there are a variety of behaviors associated with efficient flight. ${ }^{2,5,6}$

Certain body movements must be associated with unsteady aerodynamics, and the hovering we consider in this paper is of that kind. Imagine a planar wing executing a vertical flapping movement as shown in Fig. 1. If the length $A$ denotes the amplitude of the wing tip from lowest to highest point, then $f A$ is a rough indicator of the average velocity of the wing through its cycle, which will be the reference velocity; $f A \sim U$. Lift production must occur in this case through the shedding of vorticity, as the edges of the wing move through the air.

Our experiment depends on the fact that this vortex shedding can either be created by an active wing, driven by external force and torque through a flapping motion of the above kind, or else it can occur when the air is driven past the wing by an external source. We shall exploit this alternative and describe observations of a simple hovering body 


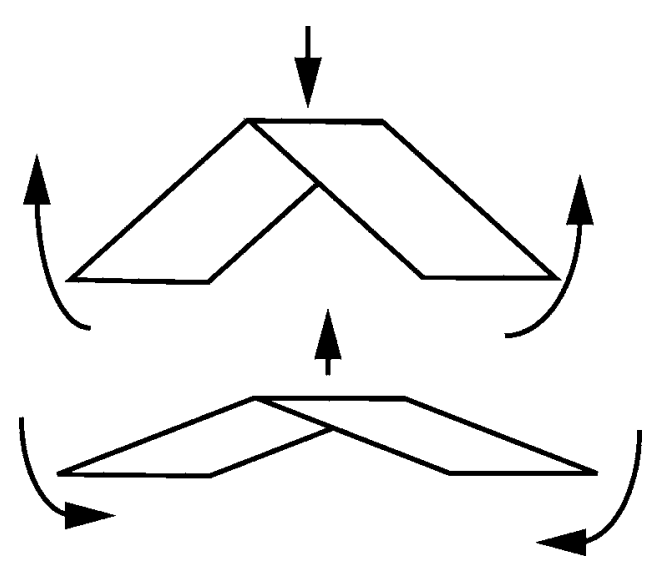

FIG. 1. A planar wing flaps actively and symmetrically about an edge. The upward movement of the wings is accompanied by a downward movement of the hinge point. The lift production is associated with vortices produced as the wing tips move up relative to the air. Similarly, on the downstroke the hinge point moves up and a second pair of vortices are produced. In each case the sense of the vortices is opposite to the arrows indicating the direction of the wing tips. The driving amplitude here is that of the wing relative to the hinge point. In our oscillating flow the driving amplitude is that of the ambient air.

situated in an oscillating air column. We shall first describe the experimental apparatus and the observations made, and consider some implications of the data. We will then relate the probable mechanism of lift production in our experiment to that of an active flapper.

The analysis of passive hovering in an oscillating flow is no easier than that of active hovering flight. To model the conditions of our experiment, we shall consider a twodimensional body consisting of two hinged, planar wings, which flap passively in an oscillating flow. By relating lift production to the relative motion of the wing tips, we are able to make some comparisons with our observational data.

\section{THE OSCILLATING FLOW CHAMBER}

The device consists of a large hi-fi speaker (a subwoofer), upright and horizontal, attached to an aluminum enclosure (a large inverted cooking pot; see Fig. 2). A diffuser within the enclosure, composed of a stack of $10-\mathrm{cm}$ straws of diameter $3 \mathrm{~mm}$, leads into a Plexiglas ${ }^{\circledR}$ test chamber of diameter $15 \mathrm{~cm}$ and height $25 \mathrm{~cm}$. Above the test chamber sits a second soda-straw diffuser. A signal generator and amplifier power the speaker, providing the desired waveform, amplitude, and frequency.

The observations described below involve oscillation frequencies $f$ in the range $10-65 \mathrm{~Hz}$. The oscillating flow "wind tunnel" is therefore not to be confused with "acoustic suspension" devices utilizing ultrasound. Of immediate concern however is the possibility of large-scale secondary flows associated with acoustic streaming. Such streaming occurs for oscillating flows confined by walls where the no-slip condition applies. It is an interesting example of a boundarylayer effect that does not vanish in the limit of zero viscosity. ${ }^{7}$ Typical streaming velocity components in the direction of the oscillating column have a velocity $\sim u_{0}^{2} / c$, where $u_{0}=A f$ is a velocity of the air column, typically $\sim 50 \mathrm{~cm} / \mathrm{s}$, and $c$ is the speed of sound. The transverse ve-

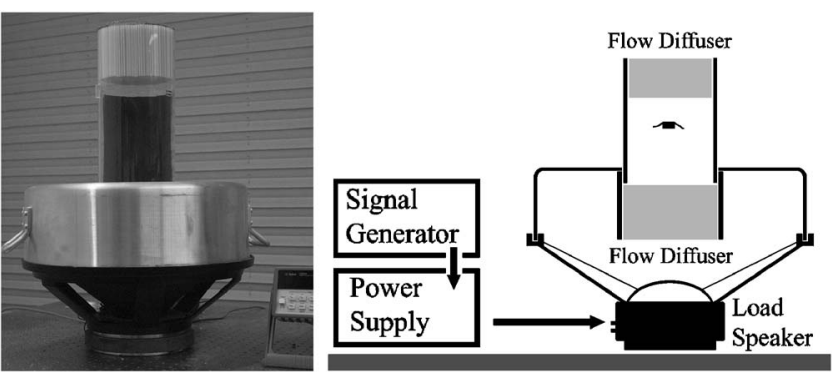

FIG. 2. A photograph and a schematic of the experimental setup. An upright loudspeaker is driven by a signal generator and amplifier.

locities are smaller by a factor $f H / c$, where $H$ is the chamber height. Thus, acoustic streaming is negligible in our experiment. That fact was confirmed by high-speed photography of the motion of a cloud (obtained by immersing dry ice in water) that was deposited at the bottom of the test section. Moreover, visualization tests using suspending particles verified the flow as essentially a bulk oscillation of the air in the test chamber.

There was, however, some contamination of the ideal column oscillation. Without the upper diffuser attached to the test chamber, there is a definite vertical asymmetry to the oscillating air column introduced by eddies shed from the edge of the test section. Nevertheless, a clean oscillation was observed in the lower part of the test section with no upper diffuser in place, and we often operated the device without it.

We remark that we were interested from the outset in the possibility that the flapping motion of the wings of a passive object might simulate the active flapping of an insect. Thus, the flexibility of the material used was paramount. In a series of trials with various papers and preparations we could see the role of flexibility on the production of lift. We found, in particular, that papers that were very stiff or geometries which could not flex at all (e.g., an inverted paper cone) could be made to hover only at extremely high amplitudes. In the present paper, we describe one set of observations using a particular preparation of the paper and geometry, and do not attempt to give quantitative results on the role of flexibility.

These hoverers, which we shall call "bugs," were constructed from thin paper. We experimented with several papers and coatings. The bugs used here were cut from tissue paper which was water-stretched on a frame and sprayed on each side with a coat of clear acrylic varnish. We used the configuration shown in Fig. 3. The wings were folded at the hinge lines to make the shape asymmetrical in the vertical, as shown in the figure.

The bug was placed in the test section and allowed to hover freely, or else a small vertical paper tube was glued to its center and slid over a vertical wire in the center of the test section, so the bug could move freely along it while hovering. We describe these cases as "free" and "tethered" hovering.

\section{OBSERVATIONS OF HOVERING}

We discuss now observations of the hovering of the bugs having the shape just described, of various diameters. At a given frequency $f$, a bug would generally begin to hover at a 


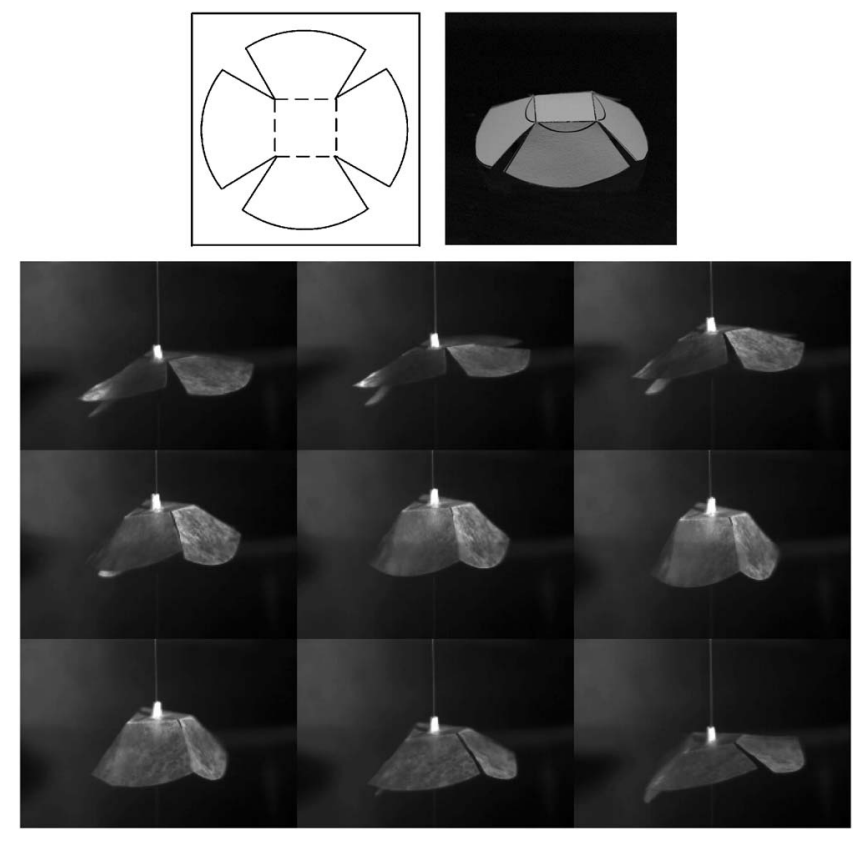

FIG. 3. Top: The planform of the bugs, and their shape in the "rest" state. Bottom: Successive frames showing a tethered bug hovering in the AC wind tunnel. The interval between frames is $6 \mathrm{~ms}$. The driving frequency is $18 \mathrm{~Hz}$. The first and last frames correspond to the lowest position of the bug. In the ascending phase the bug is open, and it closes during the descending phase.

particular amplitude $A$ of the oscillating airflow, which depended upon its size. Our measurements were performed on bugs of planform diameters $D=3,4,5$, and $6.8 \mathrm{~cm}$. (We observed hovering down to diameters of $1 \mathrm{~cm}$.) The paper used for the bugs was always the same, independent of size. In one case, $D=5 \mathrm{~cm}$ we used papers and acrylic coatings of various weights to see the effect of the increased payload. In Fig. 3, bottom, we show a sequence of images of a hovering tethered bug showing the movements of the wings in relation to the oscillation of the air.

The column air velocity has the form $U(t)=U_{0} F(2 \pi f t)$, where $F$ is approximately a sine function. The amplitude from lowest to highest position of an air particle is $A=U_{0} /(f \pi)$. For a given $f$, we found that there was a smallest value of $U_{0}$ needed to hover at a predetermined altitude above the floor of the test section. Each run consisted of two steps. We first set the frequency and determined the minimum setting of the speaker amplifier for hovering of the tethered bug. Leaving the airflow on that setting we then removed the bug and tether and sprinkled fine white powder (Vestosint 2157 particles) into the test chamber. Under proper lighting, a digital photograph was taken of the particle trace using an exposure time slightly greater than the halfperiod of the oscillation. From this image we obtained flow amplitude for several particles (about 10-20). The peak to peak amplitude of motion of the particles was uniform (within 10\%) and we used the average to estimate the flow amplitude.

We show these results in Figs. 4 and 5. In Fig. 4, for diameters $4,5,6.8 \mathrm{~cm}$, there is a clear indication of an optimal frequency for hovering as determined by the smallest

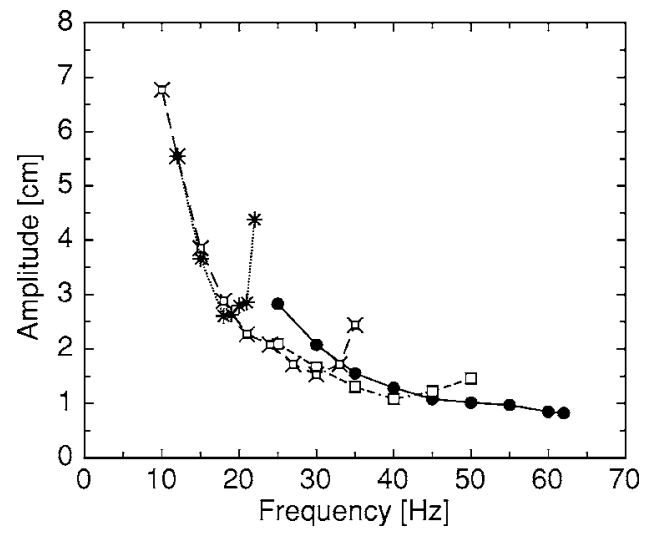

FIG. 4. Hovering boundaries for different bug sizes. For each frequency, there is a critical airflow amplitude $A$ above which bugs of diameters 3 (solid circle), 4 (square), 5 (circle-star), and $6.8 \mathrm{~cm}$ (star) hover. Amplitude is the max to min excursion of particles suspended in the flow.

airflow amplitude needed to hover. This optimal frequency increases with decreasing size for bugs of the same material. For $D=4,5$, and 6.8 , we find that at the optimal hovering frequency, the amplitude $A$ is about $0.3 D-0.4 D$. The data shown in Fig. 5 suggest that the characteristic velocity $f A$ is about the same, approximately $50 \mathrm{~cm} / \mathrm{s}$, for all bugs.

The data are revealing of the role of size in the hovering state. Although the minimum $f A$ for hovering is nearly invariant, $A$ must be independently adjusted to increase with $L$, approximately linearly $(A \approx 0.48 L-0.75 L)$. To hover efficiently, the flapping amplitude must be adjusted to bug size.

The hovering state could be made surprisingly stable by carefully constructing and folding the bug to make it very symmetric. One has the distinct impression that the flapping state has an intrinsic and quite unexpected stability. Indeed, the stability of the hovering state for a passive flexible body was the most surprising aspect of this experiment. As we have noted, the hovering configuration with wings angled downward is not a stable position for a bug falling through still air. When a bug is dropped it goes to a stable falling position with wings angled upward. The hovering state was often terminated when the bug struck the wall of the test section, the angled wings then causing it to turn over and immediately fall.

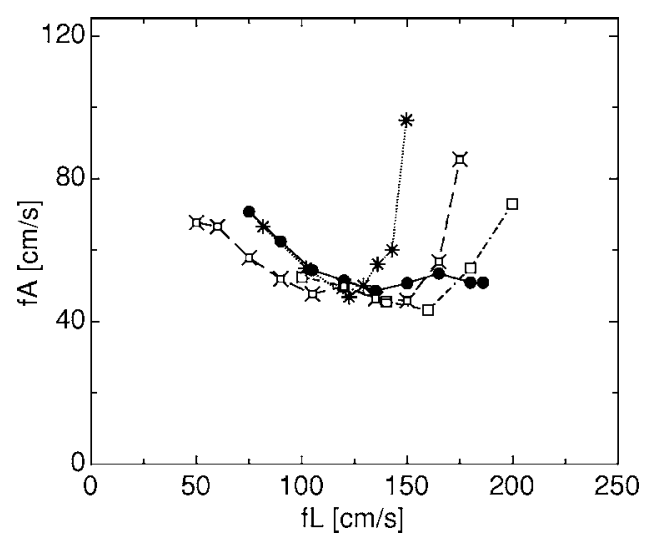

FIG. 5. $f A$ vs $f L$ for the same dataset as in Fig. 4. 


\section{ANALYSIS, SCALING, AND MECHANISMS}

Our bugs differ from those of Nature in that their weight $W$ scales with $L^{2}$ rather than $L^{3}$. A force coefficient for hovering is defined by

$$
C_{H}=\frac{W}{0.05 \rho \mathcal{U}^{2} S},
$$

where $\mathcal{U}$ is a velocity and $S$ an area. If $\mathcal{U}$ were independent of size, then $C_{H}$ should be independent of size. Figure 5 suggests that the minimum $f A$ for hovering is independent of size, but it is not obvious that $f A$ is an appropriate velocity for hovering lift, since it is the motion of the air relative to the wing that must be associated with the strength of shed vortices. To take the appropriate velocity as $U_{0}=\pi f A$ disregards the movement of the bug as well as the movement of the wing relative to the bug centroid. We photographed tethered hoverers with a high-speed movie camera and observed the movements of the wings at the critical amplitude. The center of the bug tends to move in phase with the airflow but at a smaller amplitude, $\sim D / 8$. The wings tend to move in phase but in opposition to the center of the body, with a slightly smaller amplitude, so that in fact there are only small movements of the wing tips relative to the test section. The total angular excursion of the wings is never larger than $45^{\circ}-50^{\circ}$, and thus the wings remain angled downward.

Assuming small angles and an airflow velocity $U(t)$ $=\pi f A \cos 2 \pi f t$, a reasonable formula for the velocity of the wing tip relative to the test section is

$$
u_{\text {tip }}(t)=-\frac{D \pi f}{4}(1-\gamma) \cos 2 \pi f t,
$$

where $\gamma<1$ determines the amplitude reduction of the wing relative to the airflow. With $A=0.35 D$, we see that the maximum speed of the air past the wing tip is then given by $(0.1+\gamma / 4) f \pi D$. If we take $\gamma=0.6$, we obtain the reference velocity

$$
\mathcal{U} \approx 0.25 f \pi D \approx 0.7 f \pi A \approx 110 \mathrm{~cm} / \mathrm{s} .
$$

Using this velocity in (1), taking $S$ to be the cut-out planform of the bug, and using $2.8 \mathrm{mg} / \mathrm{cm}^{2}$ for the density of the paper and $1.23 \mathrm{mg} / \mathrm{cm}^{3}$ for the density of air, we get a hovering coefficient for a typical hoverer:

$$
C_{H} \approx \frac{0.0028 \times 980}{\frac{1}{2}\left(110^{2}\right)(1.23) 10^{-3}} \approx 0.37 .
$$

Although we expect a low force coefficient for symmetric, up and down flapping movements, we point out that this $\mathcal{U}$ should actually be lower because the tip velocity is not representative of the inboard sections of the wing. In addition, if we average the velocity as sinusoid to get the rms velocity, this augments the $C_{H}$ by a factor of 2 . The essential point in these estimates is that force coefficients that are not unreasonable for insect flight have been obtained, despite the fact that we have not attempted to optimize the design of the hoverers. As Wang ${ }^{8}$ has emphasized, the customary use of the term "lift coefficient" in the case of hovering neglects the fact that the supporting force is often due to that component
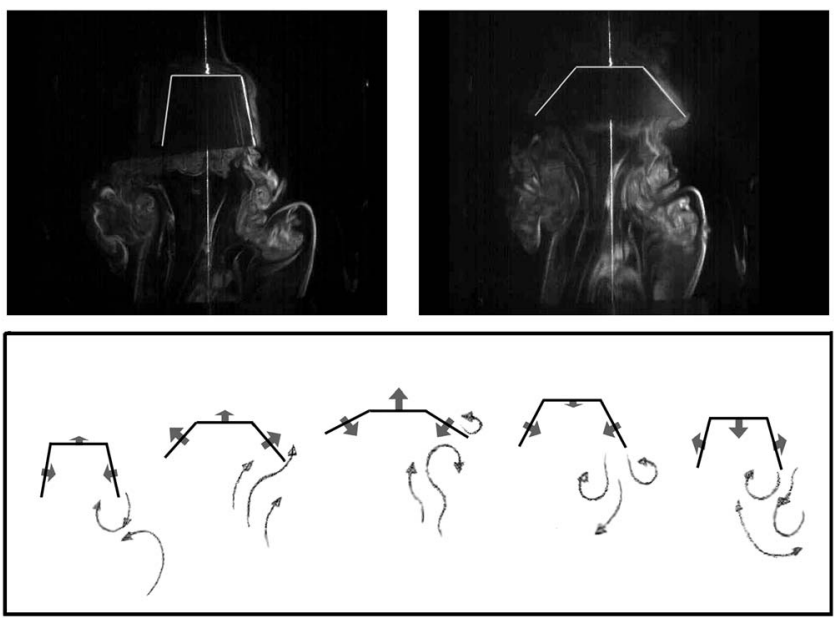

FIG. 6. Smoke visualization of the flow about the two-dimensional flapper, and the instantaneous streamlines deduced from movies of the smoke patterns around a tethered 2D flapper.

along the direction of motion, usually referred to as the drag force. We use instead the term "hovering force coefficient."

We have noted that the optimal frequency for hovering increases as the bug size is reduced. If weight is proportional to $L^{3}$ and $U=f L, S=L^{2}$, then a fixed $C_{H}$ implies that $\omega \sim L^{-1 / 2}$. In the present case, $W \sim L^{2}$ and so $f \sim L^{-1}$ by conventional scaling. For our bugs, hovering at optimal amplitudes, we have $f D=135,160,150$, and 122 for $D=3,4,5$, and 6.8 , and so this scaling is obeyed approximately. Neither scaling is obeyed by Nature's hoverers, although (for probably a variety of reasons) the wingbeat frequency of birds and insects tends to vary inversely with the size. $^{6}$

In summary, a suitable multiple of $f A$ is an appropriate velocity for defining a hovering force coefficient. Our data show that $A$ must be adjusted in proportion to $L$ to attain the force coefficient needed to initiate hovering.

\section{VISUALIZATION OF THE FLOW}

Our interest has been on the generation of lift by an oscillating flow and the relation of the lift mechanism to the hovering flight of an active flapper. We therefore attempted to visualize the shed vortices created by a tethered hoverer. Rather than deal with the three-dimensional bug geometry we inserted into our test section two parallel Plexiglas ${ }^{\circledR}$ walls separated by $1 \mathrm{~cm}$, and tethered within this section a paper bug with the cross section of the three-dimensional bug. That is, a horizontal segment was attached to two wings, the latter bent downwards to the same angle. This gave a roughly two-dimensional version of the flapper of Fig. 3. We introduced cigarette smoke into the test section and abruptly turned on the oscillating flow at an optimal amplitude and frequency. Images from the high-speed video of the smoke pattern are shown in Fig. 6. Note that the instantaneous smoke pattern does not indicate the instantaneous streamline pattern. This study was the most difficult part of our experiment, as the inevitable turbulence accompanying high Reynolds number flapping flight made the 


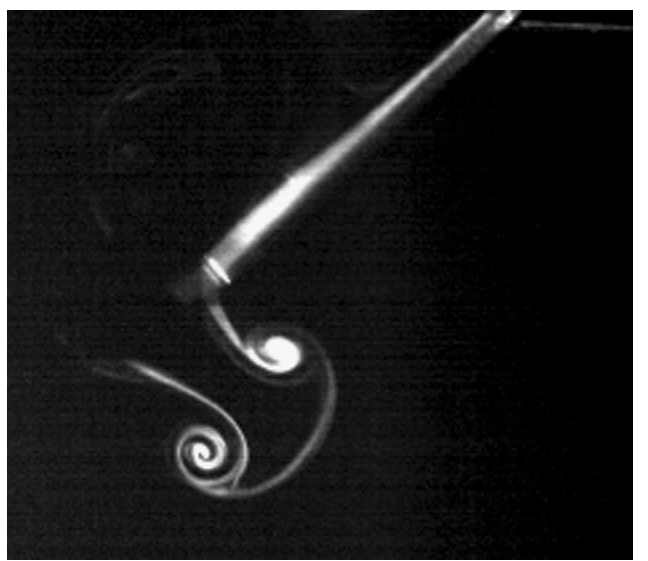

FIG. 7. Smoke visualization of the flow about a single planar wing made to move through a full up-down cycle, producing a pair of eddies.

large-scale patterns difficult to capture. Careful analysis of the movies allows the latter to be sketched, and we indicate the approximate eddy patterns in Fig. 6.

We also carried out smoke studies of a single planar wing flapped actively in still air, once through a cycle, so as to produce a pair of oppositely oriented eddies. This allowed a clear view of the eddy pair produced in active flapping (see Fig. 7).

These studies show that the mechanisms of production of vortex pairs seen in numerical simulations of active flapping are also present in the hovering of a passive flapper in an oscillating flow. We therefore propose that the passive hoverer is being supported by eddy-based momentum transport analogous, and indeed quite similar in structure, to that of an active flapper. To investigate this, we now develop a simple model based upon an estimation of the vortex shedding available in the passive case.

\section{MODELING}

The observations described in the preceding section suggest that the mechanism of hovering flight seen in our simulator is analogous to the creation of vortex pairs as observed in the two-dimensional simulations of Wang. ${ }^{9}$ Using the velocity $\pi f A \sim 150 \mathrm{~cm} / \mathrm{s}$ and a length of $2 \mathrm{~cm}$ a typical Reynolds number of our experiments is 3000 . Thus any modeling of the experiment should be at a large Reynolds number and therefore should exploit inviscid flow theory. Our object here will be to first study body motion assuming irrotationality of the flow and no separation. From the resulting flow, we will then deduce estimates for the strength of shed vortices and thereby estimate the force available for hovering. First, therefore, we shall consider a flexible, massive body, immersed in an oscillating flow field but not subjected to gravity. The body will then oscillate and deform subject to inertial forces, both from the body mass and the virtual mass of the fluid. From this study we shall deduce the movement of the wing tips relative to the distant air. This will allow a crude estimate of the circulation produced in shed eddies, and then to an estimate of the lift. Equating this lift to the body weight will then allow a qualitative study of the model which can be compared with the experimental results.

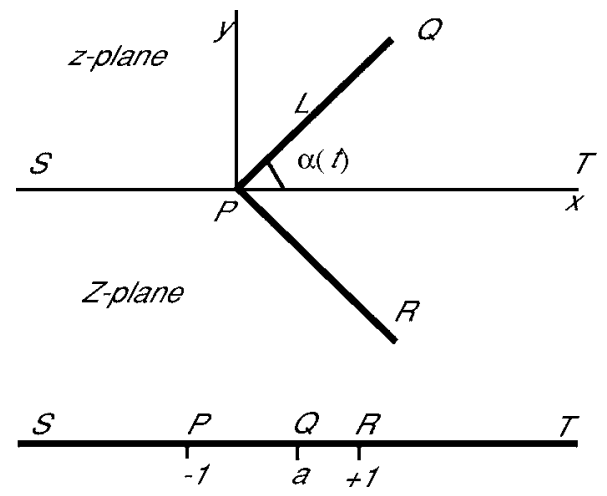

FIG. 8. A two-dimensional model of a hovering flapper.

For simplicity, we adopt the two-dimensional model shown in Fig. 8. The body will consist of two wings, each of length $L$, hinged at the point $P$ on the $x$ axis and inclined symmetrically to the horizontal with angles $\pm \alpha(t)$. (In deference to the complex notation to be used below, we orient the body so that the positive $x$ axis corresponds to the vertical direction down.) The flapping motion is symmetric about the $x$ axis. The mass of the body is taken as $m$ (per unit length), distributed uniformly along the wings. No body force is present.

We seek to determine the function $\alpha(t)$ and a position function, e.g., the $x$ coordinate of the hinge point $P$, given the ambient airflow $[U(t), 0]$, by calculating the inertial forces with no imposed body force. In the absence of vortex shedding, the flow field may be treated as irrotational and the forces and moments assumed to be purely inertial. The necessary analysis parallels a treatment of the clap and fling mechanism given by Lighthill. ${ }^{3}$ Once this flow field is known along with the motion of the body centroid and the rotation of the wings, we can compute the velocity of the wing tip relative to the oscillating air column "at infinity." We will then use this as a basis for inferring a characteristic circulation of shed vortices, and from this the force available for hovering. This is admittedly a rather crude method of estimating hovering lift, but it will allow at least a rough comparison between inviscid theory and experiment, and is appropriate here given the differences between the twodimensional (2D) and three-dimensional (3D) problems.

The 2D flapper of Fig. 8 can be conveniently analyzed in the complex $z$ plane. Our analysis will be based on standard results for locomotion in an inviscid fluid, which are summarized in the Appendix. The wings are homogeneous with a total mass (two wings) of $m$ per unit length (normal to the plane), so the centroid is located on the $x$ axis below the midpoint of the wings, and varies with both position $P$ and angle $\alpha$. However, it is acceptable to replace the centroid by any point that is fixed relative to the average body position, as it is convenient to take the hinge position $X(t)$ as the origin of the co-moving frame.

In the absence of gravity the inertial response to a periodic $U(t)=\pi A_{U} f \cos 2 \pi f t$ will be periodic and can be determined from the general analysis of the preceding section, provided that we specify how the wing angle is to be determined. We shall assume that the joint is a linear torque hinge, 
with a rest state $\alpha=\alpha_{0}$, so that the moment for deflection to an angle $\alpha$ is $k_{H}\left(\alpha-\alpha_{0}\right)$, where $k_{H}$ is a given constant. The analysis is straightforward in the general case, but is complicated by the dependence upon $\alpha$.

Modifying the analysis of Lighthill ${ }^{3}$ to account for ambient airflow, we consider the half-wing in the upper halfplane of Fig. 8. The Schwarz-Christoffel mapping from the physical $z$ plane to the upper half of the $Z=X+i Y$ plane is given by

$$
\frac{d z}{d Z}=\beta\left(\frac{Z-1}{Z+1}\right)^{\alpha / \pi} \frac{Z-a}{Z-1},
$$

where $a=1-(2 \alpha / \pi)$ is the image in the $Z$ plane of the tip $Q$ of the wing (see Fig. 8), and $\beta=L f(a)^{-1}$ with

$$
f(Z)=\int_{-1}^{Z}\left(\frac{1-u}{1+u}\right)^{\alpha / \pi} \frac{u-a}{u-1} d u .
$$

The points $Z=-1,1$ are the images of the points $P, R$ on either side of the root of the wing.

The complex potential of the flow relative to the wing root is given by

$$
w(z, t)=w_{1}(z, t)+w_{2}(z, t),
$$

where

$$
\begin{aligned}
& w_{1}(z, t)=[U(t)-\dot{X}(t)] \beta Z(z, t), \\
& w_{2}(z, t)=\frac{\dot{\alpha} \beta^{2}}{2 \pi} \int_{-1}^{+1} \frac{f^{2}(u) d u}{Z(z, t)-u} .
\end{aligned}
$$

This is equivalent to the division of potential given in the Appendix, $w_{1}$ being the complex potential of the instantaneous flow with velocity $U(t)-\dot{X}(t)$ onto the nonrotating wings, $w_{2}$ determining the effect of rotation in otherwise still air.

The $x$ force exerted by the fluid on one wing is given by $F_{x}=-\oint p d y$, where the integral is around the wing in a counterclockwise direction. In the $Z$ plane, we may extend the integral to cover the entire real axis. This force is given [cf. (A9), noting that the volume $J$ vanishes]:

$$
F_{x}=\frac{d}{d t} \Im \int_{-\infty}^{+\infty} \rho w \frac{d z}{d Z} d Z .
$$

By the residue theorem, we see that the force associated with both wings $\left(2 F_{x}\right)$ is $2 \pi \rho$ times the time derivative of the real part of the residue at $\infty$ of $w(Z) d z / d Z$. Thus, we obtain

$$
\begin{aligned}
2 F_{x}= & 2 \pi \rho \frac{d}{d t}\left\{(U-\dot{X}) \beta^{2}\left[2 \alpha / \pi-2(\alpha / \pi)^{2}\right]\right. \\
& \left.+\frac{\dot{\alpha} \beta^{2}}{2 \pi} \int_{-1}^{+1} f^{2}(u) d u\right\} .
\end{aligned}
$$

The force is balanced by the inertial force of acceleration of centroid in the absence of fluid. Since the body has mass $m$ distributed uniformly, $2 F_{x}=m\left(d^{2} / d t^{2}\right)\left(X+\frac{1}{2} L \cos \alpha\right)$ counting both wings.
As for the counterclockwise moment $M_{z}$ exerted by the fluid on the upper wing, we may again compute this from an integral along the real axis in the $Z$ plane. We have

$$
\begin{aligned}
& M_{z}=\operatorname{Re} \int p \bar{z} d z=M_{z}^{(1)}+M_{z}^{(2)}, \\
& M_{z}^{(1)}=\rho \beta^{2} \frac{\partial}{\partial t} \int_{-1}^{+1} \phi f \frac{d f}{d Z} d Z, \\
& M_{z}^{(2)}=\frac{\rho}{2} \int_{-1}^{+1}\left|\frac{d w}{d Z}\right|^{2} f \frac{d f^{-1}}{d Z} d Z .
\end{aligned}
$$

Unfortunately, for the moment, the integration path cannot be extended to $\pm \infty$ since in that case the pressure forces on the $x$ axis contribute to the moment. To simplify matters, we shall therefore make the approximation that the angle $\alpha$ differs only slightly from $\pi / 2$. In fact, to adequately model our experiment we have to accept angular displacements up to $\pi / 8$ from equilibrium, but we will accept the approximation as well within the scope and accuracy of the model.

If $\alpha$ is close to $\pi / 2$, we have $f(Z) \approx \sqrt{1-Z^{2}}, \beta \approx L$, and $X$ remains at fixed distance from the centroid. Thus, the body momentum is $m \dot{X}$ and the equation of motion is

$$
\pi \rho L^{2}(\dot{U}-\ddot{X})-m \ddot{X}+\frac{4}{3} \rho \ddot{\alpha} L^{3} \approx 0 .
$$

For the torque balance, we note that $M_{z}^{(1)}$ involves a singular integral once the contour is taken along the real axis, so that the Cauchy principal value is relevant. We then find

$$
M_{z}^{(1)} \approx \frac{d}{d t}\left[-\frac{2}{3} \rho L^{3}(U-\dot{X})-\frac{4}{3 \pi} \rho L^{4} \dot{\alpha}\right],
$$

with $M_{z}^{(2)} \approx 0$. The torque balance for the wing, taking into account the leading order inertial torques associated with rotation about the hinge point as well as motion of the hinge point, becomes

$\frac{2}{3} \rho L^{3}(\dot{U}-\ddot{X})-\frac{m L}{4} \ddot{X}+\frac{4}{3 \pi} \rho L^{4} \ddot{\alpha}+\frac{m}{4} L^{2} \ddot{\alpha}+k_{H}\left(\alpha-\alpha_{0}\right) \approx 0$.

We now let $U=\pi A f \cos 2 \pi f t, X=(B / 2) \sin 2 \pi f t, L(\alpha$ $\left.-\alpha_{0}\right)=(C / 2) \sin 2 \pi f t$. Then, (14) and (16) yield

$$
\begin{aligned}
& A-\left(1+\frac{r}{\pi}\right) B+\frac{4}{3 \pi} C=0, \\
& A-\left(\frac{3}{8} r+1\right) B+\left(\frac{2}{\pi}+\frac{3}{8} r-\frac{3}{2 K}\right) C=0 .
\end{aligned}
$$

Here the two parameters $r, K$ are defined by

$$
r=\frac{m}{\rho L^{2}}, \quad K=\frac{\rho L^{4} 4 \pi^{2} f^{2}}{k_{H}},
$$

where $r$ is the mass ratio, and $K$ is the ratio of fluid inertial forces to the elasticity of the wings. Solving these equations for $B$ and $C$ in terms of $A$ allows us to determine the wing motion given the applied flow. 
If we write this system in the form

$$
\left(\begin{array}{ll}
a & b \\
c & d
\end{array}\right)\left(\begin{array}{l}
B / A \\
C / A
\end{array}\right)=\left(\begin{array}{l}
1 \\
1
\end{array}\right)
$$

we see that for any given $L, D=a d-b c$ is positive for sufficiently small $K$ but vanishes at a unique positive value $K_{D}$. The system is singular there owing to a resonance. The effective amplitude of the oscillation of the wing tip relative to the velocity of the airflow "at infinity" is $A_{e}=A-B+C$ $=A \kappa(K, r)$, defining the function $\kappa$. Since $A_{e} / A=(D+a+b$ $-c-d) / D$, we find that $A_{e} / A$ is positive for small $K$, and again has a unique zero at a positive value $K_{A}$. Since $a+b$ $-c-d=r / \pi+2 /(3 \pi)-3 /(2 K)$, we find that $K_{A}<K_{D}$. In addition, $a-c<0$, so $C / A$ changes sign with $D$. Finally, $B / A$ is positive for small $K$ and changes sign at a point $K_{B}$ lying between $K_{A}$ and $K_{D}$. Thus, in the interval $0<K<K_{A}$, we find that $B / A>0$ and $C / A<0$; i.e., the body moves up with the air and simultaneously the wings move down, as we observe in our experiment.

We are thus in a position to discuss the relations among $f, A$, and $L$, and their bearing on hovering flight. Our calculations are based upon a simple estimate of lift generation based upon our knowledge of $A_{e}$. Each "flap" of a wing is assumed to generate a vortex with circulation $\sim A_{e}^{2} f$. The vortex pair created over one cycle establishes a momentum $\sim \rho A_{e}^{2} f L$ per unit length in a two-dimensional model, and the momentum flux per cycle is thus $\sim \rho A_{e}^{2} f^{2} L$. This force must equal the weight $m g$ in steady hovering.

For our bugs, $m$ is proportional to area, so in our twodimensional model, $m$ is proportional to $L$. It follows that $\pi A_{e} f$ should be approximately constant, independent of size, for hovering of self-similar bodies, both in our model and in our experiment. Call this constant $\mathcal{U}$. Then,

$$
\mathcal{U}=\pi f A \kappa(K, r)=A \sqrt{\frac{k_{H}}{4 \rho L^{4}}} \sqrt{K} \kappa(K, r) .
$$

Now in our experiment we took a bug of given size $L$, set $f$, and found the minimum $A$ for hovering at that $f$, producing the results shown in Figs. 4 and 5. The comparison with our model is now straightforward. For the tissue we used, $r$ is about $4.6 / L$ where $L$ is in centimeters, so setting the weight equal to $\rho \mathcal{U}^{2} L$ gives $\mathcal{U}=\sqrt{4.6 \times 980}=67 \mathrm{~cm} / \mathrm{s}$. We estimate $k_{H}=4.3$ dyn by measuring the deflection of the wing under known loading, and used $\rho=0.0012 \mathrm{~g} / \mathrm{cm}^{3}$. We are thus able to determine the hovering values of $A$ and $A f$ as functions of $L$ and

$$
f=\sqrt{\frac{k_{H}}{4 \pi^{2} \rho L^{4}}} \sqrt{K}
$$

From (21), we see that for small $K$, the $\sqrt{K}$ factor causes $A$ to diverge, as it does when $K \rightarrow K_{A}$ and $\kappa$ vanishes. Thus, there is a minimum value of $A$ in the interval $0<K<K_{A}$, just as in our frequency data. We therefore restrict the model to this last interval and plot the result in Fig. 9. Note that to put $K$ in this interval, the values of $L$ are smaller than in our experiment. This is probably a reflection of the twodimensionality of the model and the use of a simple hinge rather than a flexible sheet. A comparison of this figure with

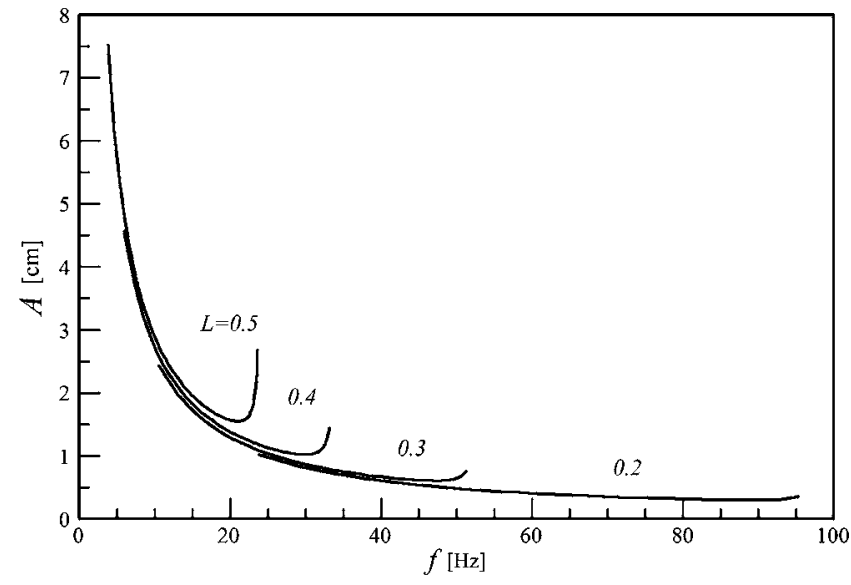

FIG. 9. $A$ vs $f$ for various $L$, for the $2 \mathrm{D}$ model and the parameters of the experimental tissue. $L$ and $A$ are in centimeters.

Fig. 4 reveals that model is surprisingly faithful in many respects. However, in our experiment, as shown in Fig. 5, we observe a clearly defined minimum of $f A$ as a function of $f L$, whereas when we compute this function in the model we do not. The difficulty here may be traced to the crude estimate of lift derived simply from wing tip velocity. In fact, lift production depends not only upon the strength of eddies, but the exact timing and position of their creation. We therefore suggest that the flexibility of the wing is important in determining the release point of eddies, in a sense taking over the delicate task of monitoring the release point of eddies of the active flapper.

Now it is easy to show that the wing angle amplitude $C$ vanishes at zero frequency. (It is decreasing approximately linearly with $K$ for positive $K$.) Consequently the model is producing finite $\mathcal{U}$ and hence finite lift at $K=0$. We have noted earlier that a nonflexing wing produces little if any lift.

Clearly, to remedy this defect we need a more accurate estimate of usable lift derived from wing tip motion. As a somewhat arbitrary correction factor, we replace the function $\kappa(K, r)$ by $\left|\left(C / A_{e}\right)\right|^{1 / 2} \kappa(K, r)$. This produces zero lift at $K=0$ and leads to the curves shown in Fig. 10, which resemble reasonably well the experimental curves. The lower

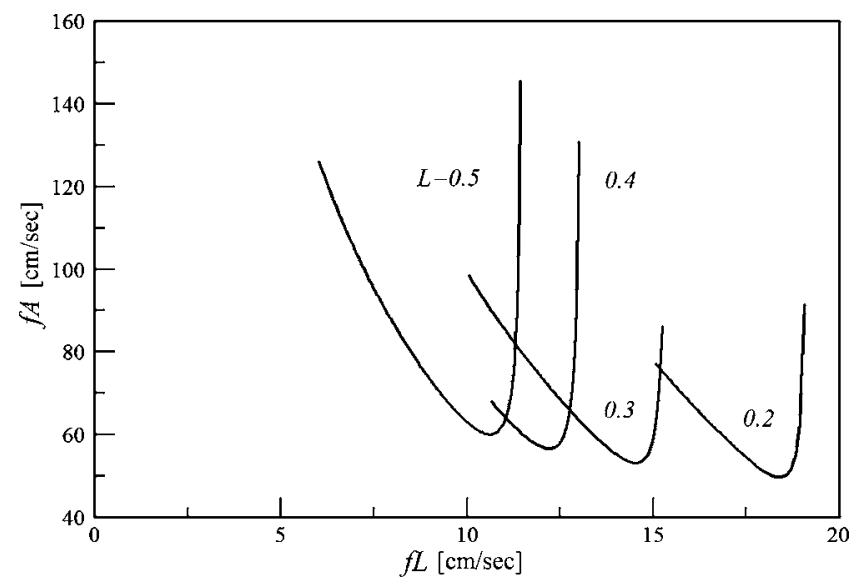

FIG. 10. Model results using the data from Fig. 9, but including a lift cutoff at small $K$. 
values of $f L$ in the model, by an order of magnitude, are thought to reflect the substantially different geometries of the 2D model and the 3D bugs.

The model correctly tracks the increase of $A$ and $f A$ for large $K$, because this is a result of the inertial lag of the wing tip, reducing the effective tip velocity. In fact, a singularity in $A$ and $f A$ occurs when the tip velocity vanishes. For still larger $f, A_{e}$ becomes infinite and our model cannot be valid.

\section{CONCLUDING REMARKS}

The primary object of this paper has been to demonstrate an experimental tool for the study of hovering flight. We have used the term "simulator" to stress the fact that unsteady aerodynamics arising from the shed vorticity can apparently account for the passive hovering flight we observe. The mechanisms are believed to be very similar to those of an active hoverer, but further flow visualization studies are needed to verify this proposal. In particular a careful analysis of the paired vortices produced, as a function of frequency, might indicate the proper low-frequency cutoff of the lift.

A surprising feature of the device is the stability of the hovering state. We are unaware of any study of the stability of a free passive flapper, but our observations suggest that for the passive bodies there is an inherent and quite unexpected stability induced by the unsteady vortical field, and perhaps also by the flexibility. It is not clear, of course, that an inherent stability is achieved or is even desirable in natural flight. As a rule one accepts loss of stability for increased maneuverability. However for artificial, robotic hoverers, where the main object is to provide a stable observational platform, stability of free hovering is desirable.

There are undoubtedly many "bug" geometries that can improve on both stability and lift production. The examples studied here were chosen primarily for simplicity and ease of construction. In preliminary studies of bugs with asymmetric geometry, Huebscher found wing planforms that caused rotation of the bug simultaneously with the flapping, and this enhanced stability. This raises the possibility of increasing lift and stability by constructing "flapping helicopters."

Despite the observed stability, it was not possible in our device to fix the flapper in place without using a tether. This was because of turbulence in the flow as it moved up and down through the diffusers. Further studies are needed to suggest how the airflow might be modified to reduce motion of the bug and provide better conditions for flow visualization.

Although the theory given here is simple and approximate, it is the appropriate first step for comparing motion of air past a wing as opposed to moving the wing through the air. We have not adequately accounted for that variation of lift with the amplitude which is due to the changing release points of the shed vortices. An improved theory would presumably dispense with the ad hoc cutoff of lift at small frequencies. One might, for example, incorporate the direct numerical computation of shed vortex sheets in a 2D model of the kind studied here. ${ }^{10}$

\section{ACKNOWLEDGMENTS}

We thank Sunny Jung and Nathanial Huebscher for their help with many aspects of the experiment. The research reported in this paper was supported by the National Science Foundation under Grants No. DMS-9980069 and No. DMS0507615 at New York University, and by the Department of Energy under Grant No. DE-FG0288ER25053 at New York University.

\section{APPENDIX: LOCOMOTION IN AN INVISCID FLUID}

We give here a few general results concerning locomotion of time-dependent bodies in an inviscid fluid, ${ }^{11,12}$ but including a time-dependent ambient flow. For simplicity we assume that no body force is present, and that the body is homogeneous and of constant volume. Let the position of the centroid be $X(t)$ in the co-moving frame. If the ambient flow is $\mathbf{U}(t), p=-\rho \mathbf{x} \cdot \dot{\mathbf{U}}$, we can compute the disturbance flow created by a free time-dependent body by moving to a rest frame in which the velocity of the fluid at infinity is zero. We will also want to consider a co-moving frame where the centroid remains fixed.

Relative to the rest frame we may divide up the potential flow into two parts; i.e., $\phi^{\prime}=\phi_{1}^{\prime}+\phi_{2}$. The first satisfies the condition that

$$
\frac{\partial \phi_{1}^{\prime}}{\partial n}=\mathbf{n} \cdot(\dot{\mathbf{X}}-\mathbf{U})
$$

on the body surface; say, $S(t)$. Thus, $\phi_{1}^{\prime}$ accounts for the translation of the body through the ambient air. $\phi_{2}$ must then account for the time dependence of the body relative to its centroid, for which we assume a given normal velocity component $v_{S}(t)$ defined on the surface $S$. Thus,

$$
\frac{\partial \phi_{2}}{\partial n}=v_{S} .
$$

Both $\phi_{1}^{\prime}$ and $\phi_{2}$ decay at least as fast as $\left(x^{2}+y^{2}+z^{2}\right)^{-1}$ at infinity.

Relative to the co-moving frame, the flow field has the potential

$$
\phi=\phi_{1}+\phi_{2}, \phi_{1}=\mathbf{x} \cdot(\mathbf{U}-\dot{\mathbf{X}})+\phi_{1}^{\prime} .
$$

Note that $\partial \phi_{1}^{\prime} / \partial n=0$ on the body surface.

We shall now consider the force $\mathbf{F}$ exerted by the fluid on the body. Computing this in the co-moving frame, we note that the pressure is then given by

$$
p=-\rho \mathbf{x} \cdot(\dot{\mathbf{U}}-\ddot{\mathbf{X}})-\rho\left[\phi_{t}+\frac{1}{2}(\nabla \phi)^{2}\right]
$$

by the unsteady Bernoulli theorem for irrotational flow. Thus,

$$
\mathbf{F}=\rho J(\dot{\mathbf{U}}-\ddot{\mathbf{X}})+\rho \int_{S} \phi_{t} \mathbf{n} d S+\frac{1}{2} \rho \int_{S}(\nabla \phi)^{2} \mathbf{n} d S,
$$

where $J$ is the (constant) body volume, and $\mathbf{n}$ is the outward normal to $S$. Now the last term may be written, using subscript notation and after applying the divergence theorem, 


$$
\begin{aligned}
& \frac{1}{2} \rho \int_{S}\left(\nabla \phi_{1}\right)^{2} n_{i} d S-\rho \int \frac{\partial}{\partial x_{k}} \\
& \quad \times\left[\frac{\partial \phi_{1}}{\partial x_{i}} \frac{\partial \phi_{2}}{\partial x_{k}}+\frac{\partial \phi_{2}}{\partial x_{i}} \frac{\partial \phi_{2}}{\partial x_{k}}+\frac{\partial \phi_{1}}{\partial x_{k}} \frac{\partial \phi_{2}}{\partial x_{k}}\right] d V .
\end{aligned}
$$

In addition, a theorem of the calculus involving material volumes yields

$$
\begin{aligned}
\int_{S} \phi_{t} n_{i} d S & =-\int_{V} \frac{\partial}{\partial t} \frac{\partial \phi}{\partial x_{i}} d V \\
& =-\frac{\partial}{\partial t} \int_{V} \frac{\partial \phi}{\partial x_{i}} d V-\int_{S} v_{S} \frac{\partial \phi}{\partial x_{i}} d S
\end{aligned}
$$

Combining these terms, our expression for the force $\mathbf{F}$ may be written

$$
\mathbf{F}=\rho J(\dot{\mathbf{U}}-\ddot{\mathbf{X}})+\rho \frac{\partial}{\partial t} \int_{S} \phi \mathbf{n} d S+\frac{1}{2} \rho \int_{S}\left(\nabla \phi_{1}\right)^{2} \mathbf{n} d S .
$$

The last term on the right of (A8) is the pressure force of translation of a rigid body in irrotational flow, which is zero (d'Alembert's paradox). Thus, the inertial force is given by

$$
\mathbf{F}=\rho J(\dot{\mathbf{U}}-\ddot{\mathbf{X}})+\rho \frac{\partial}{\partial t} \int_{S} \phi \mathbf{n} d S .
$$

By Newton's law, $F=m \ddot{\mathbf{X}}$, where $m$ is the mass of the body.
A similar argument applies to the moment on a body subject to no external torques. Omitting the details, the result is that the moment $\mathbf{M}$ exerted by the fluid on the body is given by

$$
\mathbf{M}=\rho \frac{\partial}{\partial t} \int_{S} \phi \mathbf{x} \times \mathbf{n} d S+\frac{1}{2} \rho \int_{S}\left(\nabla \phi_{1}\right)^{2} \mathbf{x} \times \mathbf{n} d S
$$

and this moment must equal the time derivative of angular momentum of the body.

${ }^{1}$ C. Ellington, "The aerodynamics of hovering insect flight IV," Philos. Trans. R. Soc. London, Ser. B 305, 1 (1984).

${ }^{2}$ Z. J. Wang, "Dissecting insect flight," Annu. Rev. Fluid Mech. 37, 183 (2005).

${ }^{3}$ M. J. Lighthill, Mathematical Biofluiddynamics, in Regional Conference Series in Applied Mathematics Vol. 17 (Society for Industrial and Applied Mathematics, Philadelphia, 1975).

${ }^{4}$ Z. J. Wang, J. M. Birch, and M. H. Dickinson, "Unsteady forces and flows in low Reynolds number hovering flight: two-dimensional computations vs. robotic wing experiments," J. Cell Physiol. 207, 449 (2004).

${ }^{5} \mathrm{M}$. Dickinson, "Unsteady mechanisms of force generation ins aquatic and aerial locomotion," Am. Zool. 36, 537 (1996).

${ }^{6} \mathrm{R}$. Dudley, The Biomechanics of Insect Flight (Princeton University Press, Princeton, 2000).

${ }^{7}$ L. Landau and E. Lifshitz, Fluid Mechanics, in Course of Theoretical Physics Vol. 6 (Pergamon, New York, 1987).

${ }^{8}$ Z. J. Wang, "The role of drag in insect hovering," J. Cell Physiol. 207, 4147 (2004).

${ }^{9}$ Z. J. Wang, "Two dimensional mechanism for insect hovering," Phys. Rev. Lett. 85, 2216 (2000).

${ }^{10} \mathrm{M}$. A. Jones, "The separated flow of an inviscid fluid around a moving flat plate," J. Fluid Mech. 496, 405 (2003)

${ }^{11} \mathrm{P}$. Saffman, "The self-propulsion of a deformable body in a perfect fluid," J. Fluid Mech. 28, 385 (1967).

${ }^{12}$ S. Childress, Mechanics of Swimming and Flying (Cambridge University Press, Cambridge, 1981). 